\title{
The Risk of Venous Thromboembolism (VTE) in Men with Benign Prostatic Hyperplasia Treated with 5-Alpha Reductase Inhibitors (5ARIs).
}

\author{
Olulade Ayodele' \\ Howard J Cabral ${ }^{2,3}$ \\ David McManus ${ }^{4}$ \\ Susan Jick $\mathbb{D}^{1,5}$
}

'Department of Epidemiology, Boston University School of Public Health, Boston, MA, USA; ${ }^{2}$ Department of Biostatistics, Boston University School of Public Health, Boston, MA, USA;

${ }^{3}$ Biostatistics and Research Design Program, Boston University Clinical and Translational Science Institute, Boston, MA, USA; ${ }^{4}$ Department of Medicine, University of Massachusetts Medical School, Worcester, MA, USA; ${ }^{5}$ Boston Collaborative Drug Surveillance Program, Lexington, MA, USA
Correspondence: Susan Jick Boston Collaborative Drug Surveillance Program, Lexington, MA, USA Tel +l 781 862-6660

Email sjick@bu.edu
Background: Many men receive 5-alpha reductase inhibitors (5ARIs) for ongoing treatment of benign prostatic hyperplasia (BPH). The increased risk of cardiovascular complications with 5ARIs has been documented in BPH studies and the occurrence of cerebral venous thrombosis, presumably due to increased estrogen level following 5ARI use, was described in multiple case reports. The objective of this study was to determine if 5ARIs with or without alpha blockers $(\mathrm{AB})$ were associated with an increased risk of venous thromboembolism (VTE) in males with BPH.

Methods: We conducted a nested case-control study among a population of men ages 40 79 who received at least one 5ARI or AB prescription for treatment of BPH between 1995 and 2015 in the UK-based Clinical Practice Research Datalink GOLD. Cases of incident VTE (pulmonary embolism [PE] or deep venous thrombosis [DVT]) and matched controls were identified from this population. We used descriptive analyses and conditional logistic regression to evaluate the risk of VTE in users of 5ARIs compared to users of ABs.

Results: For 5ARI only users, the adjusted odds ratios (aORs), (95\% CI) for VTE were 1.51 (0.98-2.32) in current 5ARI users and $1.23(0.70-2.17)$ in recent/distant past, compared to $\mathrm{AB}$ only users. However, the aOR $(95 \% \mathrm{CI})$ in men who had 50 or more current $5 \mathrm{ARI}$ prescriptions compared to users of ABs only was higher: 2.29 (1.14-4.63). For 5ARI with $\mathrm{AB}$ use, the aORs, $(95 \% \mathrm{CI})$ for VTE were $1.16(0.64-2.10)$ in current $5 \mathrm{ARI}+\mathrm{AB}$ users and $1.93(0.71-5.25)$ in recent/distant past, compared to AB only users. The aOR $(95 \% \mathrm{CI})$ in men who had 50 or more current $5 \mathrm{ARI}+\mathrm{AB}$ prescriptions compared to users of $\mathrm{ABs}$ only was 1.65 (0.64-4.26).

Conclusion: Current use of 5ARI, particularly long-term use, is associated with an increased risk of incident idiopathic VTE compared to patients treated with AB use only.

Keywords: 5ARI, BPH, VTE, alpha blocker

\section{Introduction}

Benign prostatic hyperplasia (BPH) is a highly prevalent condition among older men, which impacts quality of life, sexual function, and genitourinary health. Close to $30 \%$ of men over the age of 50 have $\mathrm{BPH}^{1,2}$ The two main drug classes prescribed for management of BPH symptomatic relief are alpha-blockers (ABs) and 5-alpha reductase inhibitors (5ARIs) either alone or in combination. ${ }^{2,3}$

To the best of our knowledge, this is the first study to evaluate the risk of venous thromboembolism (VTE) in relation to the use of 5ARIs. The closest comparisons available are in studies that evaluated the risk of VTE and cardiovascular outcomes 
associated with testosterone use and androgen deprivation therapy. Studies of cardiovascular safety in men who take 5ARIs are limited and have yielded conflicting results. While some studies suggest dihydrotestosterone (DHT) reduces cardiovascular disease risk, others suggest that androgen (DHT and testosterone) suppression increases the incidence of myocardial infarction (MI), stroke and diabetes. ${ }^{4-7}$ Other studies found an increased short-term risk of VTE in testosterone users. ${ }^{8}$ Since 5ARIs prevent the formation of DHT, the active form of testosterone, 9,10 it is possible that 5ARIs decrease the risk of developing VTE. Multiple case reports have indicated 5ARI as a potential cause of cerebral venous thrombosis potentially due to increased serum estrone and estradiol levels. ${ }^{11,12}$ Similarly, multiple studies have found an increased risk of gynecomastia among users of 5ARIs, ${ }^{13-25}$ possibly due to an increase in estrone and estradiol levels. ${ }^{9,26-28}$ These hormones promote thrombus development and can thus increase VTE risk. ${ }^{29-32}$

5ARIs have become widely used for management of $\mathrm{BPH}$; thus, it is important to understand the potential longterm adverse or beneficial effects of their use in this population that is typically older, has multiple comorbidities and many known VTE risk factors that could confound the 5ARI-VTE relationship. To our knowledge, these hormonal effects are not anticipated with ABs. In this study, we evaluated the effects of 5ARIs compared to an active comparator group, $\mathrm{AB}$ use (to control for confounding by indication), on the risk of VTE in a population-based sample of men aged 40-79 with BPH in the United Kingdom.

\section{Methods}

This was a nested case-control study conducted using Clinical Practice Research Datalink (CPRD) GOLD data from years 1995-2015. CPRD GOLD is an ongoing longitudinal database, established in 1987, of over 400 general practices in the UK. It contains data on a representative sample of more than 10 million UK patients recorded by general practitioners (GPs) using standard software and coding systems. In addition to patient demographic information, participating GPs record anonymized data that includes medical diagnoses, prescriptions issued in primary care (including dosage and quantity), details of hospital stays and specialist referrals, laboratory tests, lifestyle details [such as smoking, body mass index (BMI), alcohol use] and deaths. Large validation studies have demonstrated the accuracy and completeness of data captured in the CPRD GOLD for use in pharmacoepidemiologic research. ${ }^{33-35}$ This study is based in part on data from the CPRD obtained under license from the UK Medicines and Healthcare products Regulatory Agency. The data is provided by patients and collected by the NHS as part of their care and support. This study was approved by the Independent Scientific Advisory Committee (ISAC) for Medicines and Healthcare Products Regulatory Agency database research (protocol no: $17 \_148 \mathrm{R}$ ), and the protocol was made available to the journal reviewers upon request.

The study population was comprised of 91,115 men aged 40-79 in CPRD GOLD, who had a diagnosis of BPH identified using Read codes and who had received at least one prescription for a 5ARI (finasteride or dutasteride) and/or an $\mathrm{AB}$ (alfuzosin, doxazosin, indoramin, prazosin, tamsulosin and terazosin) for the treatment of BPH during 1995-2015.

\section{Outcome}

Cases were men with an incident idiopathic VTE [Read code for Deep Venous Thrombosis (DVT) or Pulmonary Embolism (PE)] after the electronic medical record start date. We required all cases to have one or more years of information in the CPRD before the index date, defined as the date of the first VTE diagnosis in the cases or the matched date for controls. Cases with cancer (except nonmelanoma skin cancer) or history of VTE prior to the index date were excluded. Idiopathic VTE was defined as VTE in the absence of a code for another proximate cause (including immobility, prolonged hospitalization, fractures, multiple traumas, orthopedic surgery involving long bones or pelvis and, other major surgery) within 90 days prior to the first VTE diagnosis. Cases were required to have 2 or more prescriptions for anticoagulants (including unfractionated heparin, low-molecular-weight heparins and fondaparinux) present on or within 90 days after the VTE diagnosis date to increase the specificity of the case definition.

\section{Control Selection}

From the base population, we matched up to four controls to each eligible case on index date (same index date as their matched case), year of birth ( \pm 3 years), general practice attended and the year the patient's CPRD record started ( \pm 3 years). The same inclusion and exclusion criteria used in cases were applied to controls. 


\section{Exposure- 5ARI and $A B$ Use}

A patient was considered exposed if they had at least one prescription for a 5ARI in their record prior to the index date. Patients were assigned to one of the following mutually exclusive exposure categories based on the closest prescription prior to the index date: 5ARI only, or $5 \mathrm{ARI}+\mathrm{AB}$ (on the same date). We also evaluated 5ARI exposure according to timing of use: We categorized exposure as current use of 5ARI if the length of the last prescription continued to the index date (where use was defined as the prescription length +30 days). Recent use was defined as use that ended between 30 and 119 days prior to the index date. Past use was defined as use that ended between 120 days and less than 305 days prior to the index date, and distant past use was defined as use that ended 305 days or more prior to the index date.

In addition, we examined the number of 5ARI prescriptions recorded before the index date $(0,1-6,7-24$, $25-49,50+)$ and the total cumulative dose of 5ARI in micrograms recorded before the index date $(0,1-999$, $1000-9999,10,000+)$ in current users, to assess whether heavier use was associated with elevated risk of VTE. To calculate the cumulative dose for each study participant, we multiplied the number of pills in each prescription by their strength and summed all prescriptions before the index date.

The referent group was comprised of patients whose most recent prescription prior to the index date was for an $\mathrm{AB}$ only, regardless of how long before the index date it was received.

\section{Covariates}

Information on covariates was derived from the patient records and included known or suspected risk factors for VTE: age, smoking status (never, past, current and unknown), calendar time (to capture secular trends in VTE risk and 5ARI or AB use), BMI $(<24.9,25-29.9$, $\geq 30 \mathrm{~kg} \mathrm{~m}-2$, unknown) and comorbidities- (including diabetes [with/without end-organ damage], chronic kidney disease (CKD), cerebrovascular disease, cardiovascular disease [MI, heart failure, peripheral vascular disease], chronic obstructive pulmonary disease, dementia, hemiplegia, peptic ulcer disease, acquired immune deficiency syndrome, liver disease, connective tissue disease including vasculitis, AIDS, alcohol abuse disorders, hypertension, coagulopathies, and phlebitis). We defined each comorbidity based on the presence of at least one Read code prior to the index date. We then calculated a Charlson Comorbidity Index (CCI) score for each patient ${ }^{36,37}$ and categorized them into 3 groups: $1-3,4$ and $5+$.

Finally, we classified each case and control by whether they switched between the study drugs (ABs and 5ARIs) before the index date (yes/no) and by the duration of BPH prior to the index date $(<5$ years and 5 + years).

\section{Data Analysis}

We calculated crude and adjusted odds ratios (OR) and $95 \%$ confidence intervals $(95 \% \mathrm{CI})$ to estimate the risk of VTE in patients ever and currently exposed to 5ARIs (with or without $\mathrm{AB}$ ) at the index date in comparison with patients ever exposed to $\mathrm{AB}$ only using conditional logistic regression modelling for matched pairs using PROC LOGISTIC using SAS statistical software (version 9.4, Cary, NC: SAS Institute. Inc).

We evaluated covariates that were structurally deemed to be confounders, based on directed acyclic graphs (history of phlebitis, diabetes, COPD, CVD, obesity, smoking, CKD, hyperlipidemia, CCI score) to determine if they were confounders of the 5ARI-VTE association. Each covariate was added to the model one at a time, and the relationship with the exposure and outcome was assessed. If the addition of the covariate altered the measure of association by at least $10 \%$, it was included in the adjusted model. The final model included BMI, CKD, switching between 5ARIs and ABs, cardiovascular and cerebrovascular disease separately and all other comorbidities were summarized by CCI scores. We stratified the results by age, BPH duration, switching between $\mathrm{AB}$ and 5ARI, obesity status and CCI score to assess effect measure modification (EMM).

We assessed the potential for residual confounding in sensitivity analyses restricted to "healthy" cases and controls (ie those with no diagnosis for VTE related comorbidities such as cerebrovascular disease, coronary heart disease, heart failure, COPD, autoimmune disorders, diabetes and hypertension prior to the index date). We also conducted sensitivity analyses where we defined current use as use that ended within 14 days, recent: 15-119 days, past: 120-305 days and distant past: $>305$ days. Finally, we also conducted sensitivity analyses where we compared 5ARI exposure to $A B$ only by recency of use, ie current, recent or past $A B$ use, instead of any use. 


\section{Results}

We identified 266 idiopathic VTE cases who met all study criteria to which we matched 1061 controls. There were 64 (24\%) cases and 218 (21\%) controls exposed to a 5ARI only at the index date, $33(12 \%)$ cases and $130(12 \%)$ controls exposed to a $5 \mathrm{ARI}+\mathrm{AB}$ and $169(64 \%)$ cases and 713 (67\%) controls exposed to an $\mathrm{AB}$ only at the index date.

The distribution of potential VTE risk factors and patient characteristics, as well as the bivariable OR estimates and associated 95\% CIs, are presented in Table 1. VTE cases were more likely than controls to have a history of cardiovascular disease, chronic kidney disease, phlebitis, COPD, smoking and a higher body mass index (BMI $>30+$ ). Sixty-two percent of cases had at least one comorbidity compared with $56 \%$ of controls and $37 \%$ of cases had a Charlson comorbidity index score $\geq 5$ compared with $31 \%$ of controls.

5ARI users were older, more likely to have cardiovascular and renal disease, and to have a longer BPH duration compared to $5 \mathrm{ARI}+\mathrm{AB}$ users or $\mathrm{AB}$ only users. See Table 2.

The OR of VTE was slightly elevated in ever users of 5ARIs only (adjusted OR [aOR] 1.41 (95\% CI 0.97-2.07)) and ever users of $5 \mathrm{ARI}+\mathrm{AB}(\mathrm{aOR} 1.27,95 \%$ CI $0.72-$ 2.21) compared to $A B$ only users. When we evaluated current 5ARI use, the risk of VTE was slightly higher (aOR 1.51, 95\% CI 0.98-2.32) compared to $\mathrm{AB}$ only use. The numbers were small in all analyses and all CIs included 1.0. The OR for current use of 5ARI $+\mathrm{AB}$ compared to $\mathrm{AB}$ only use and for non-current use of 5ARIs only remained close to the null (aOR $=1.16,95 \%$ CI $0.64-2.10)$, and (1.23, 95\% CI 0.70-2.17), respectively (Table 3). In the models where we evaluated current 5ARI use, with or without concurrent $\mathrm{AB}$ use, the results were attenuated $(\mathrm{aOR}=1.38(0.93-2.06))$ compared to models with 5ARI use alone versus $\mathrm{AB}$ (Table 4).

When we classified 5ARI exposure by number of prescriptions and cumulative dose to evaluate the presence of a dose response relationship, the risk of VTE was highest in the highest use categories (Table 5): $\mathrm{aOR}=2.29,95 \% \mathrm{CI}$ 1.14-4.63 for current 5ARI users with 50 or more prescriptions and $\mathrm{aOR}=2.15,95 \%$ CI $1.18-3.92$ for cumulative dose of 10,000 mcg and higher compared to $\mathrm{AB}$ users.

In stratified analyses, there was no evidence of effect modification by age [Supplement 1].

We evaluated BMI as a potential mediator on the causal pathway between 5ARI use and VTE. However, we did not observe any material change or reduction in the effect size between the crude OR (OR Current 5ARI only use $=1.36,95 \%$ CI $0.92-2.03)$ and the BMI adjusted OR (OR Current 5ARI only use $=1.39,95 \%$ CI $0.93-2.07$ ). We observed an increased risk of VTE $(\mathrm{aOR}=2.7,95 \% \mathrm{CI}$ 1.1-6.7) in current 5ARI only users who were obese $\left(\mathrm{BMI} \geq 30 \mathrm{~kg} / \mathrm{m}^{2}\right)$ compared to $\mathrm{AB}$ users [Supplement 2] but the cell sizes were small. For men who were not obese $\left(\mathrm{BMI}<30 \mathrm{~kg} / \mathrm{m}^{2}\right)$, the effect size was smaller $(\mathrm{aOR}=1.4$, 95\% CI 0.9-2.2), and the result is consistent with a null effect. The pattern was similar in models where we evaluated the number and cumulative dose of 5ARI prescriptions.

We evaluated effect measure modification by $\mathrm{CCI}$ score. Due to the small cell sizes, we evaluated any current 5ARI use (ie with or without concurrent $\mathrm{AB}$ use) compared to $A B$ use. The risk of VTE increased with higher comorbidity scores (test for trend $\mathrm{p}=0.01$ ). For current users of $5 \mathrm{ARI} \pm \mathrm{AB}$ compared to $\mathrm{AB}$ use, the aOR of VTE was $1.38,95 \%$ CI $(0.93-2.06)$; for men with a CCI score of $1-3$ the aOR was $0.94,95 \%$ CI $(0.48-1.82)$; for men with a score of 4 the aOR was 1.53 , (95\% CI $0.71-$ 3.28 ) and for men with a score of $5+$ the aOR was 1.57 (95\% CI 0.90-2.75) [Table 4].

Redefining current use in a sensitivity analysis as 5ARI use that ended within 14 days (instead of 30) of the index date did not materially change the magnitude of the effect estimates [Supplement 3].

There was no material difference in results when we conducted sensitivity analyses to assess current 5ARI use compared to current $\mathrm{AB}$ use [Supplement 4], nor were the results meaningfully different from the main analysis when we conducted sensitivity analysis restricted to the VTE cases with no history of CKD, vasculitis or coagulopathy (adjusted OR 1.66, 95\% CI 0.97-2.83 for current 5ARI use and $1.39,95 \% \mathrm{CI} 0.70-2.76$ for current $5 \mathrm{ARI}+\mathrm{AB}$ use, compared to $\mathrm{AB}$ ) [data not shown], or when we excluded cases with diagnoses of hypertension, diabetes, hyperlipidemia, cerebrovascular disease, coronary heart disease, heart failure, COPD, autoimmune disorders, and severe liver disease prior to the index date.

\section{Discussion}

The results of our study on this population of men aged 40-79 years with treated BPH suggest that current 5ARI use, particularly at high cumulative dose, is associated with an increased risk of incident idiopathic VTE when compared to patients treated with $\mathrm{ABs}$ only; aOR for any 
Table I Characteristics and Bivariate Odds Ratios in Venous Thromboembolism Cases and Matched Controls; BPH Patients Aged 40-79 in CPRD GOLD 1995-2015

\begin{tabular}{|c|c|c|c|c|}
\hline & Cases $(n=266)$ & Controls $(n=1061)$ & OR & $95 \% \mathrm{Cl}$ \\
\hline \multicolumn{5}{|l|}{ Age at Index Date (years) } \\
\hline$<60$ & $23(8.6)$ & $87(8.2)$ & $N A^{a}$ & $N A^{a}$ \\
\hline $60-69$ & $66(24.7)$ & $267(25.1)$ & $N A^{a}$ & $N A^{a}$ \\
\hline $70-74$ & $60(22.5)$ & $233(21.9)$ & $N A^{a}$ & $N A^{a}$ \\
\hline$\geq 75$ & II 8 (44.2) & $478(44.9)$ & $N A^{a}$ & $N A^{a}$ \\
\hline Mean \pm Std. Dev & $73.2 \pm 8.4$ & $73.2 \pm 8.3$ & $\mathrm{NA}^{\mathrm{a}}$ & $\mathrm{NA}^{\mathrm{a}}$ \\
\hline \multicolumn{5}{|l|}{ Index Year } \\
\hline $1995-1999$ & $6(2.3)$ & $24(2.3)$ & $N A^{a}$ & $N A^{a}$ \\
\hline $2000-2004$ & 37 (I3.9) & $148(13.9)$ & $N A^{a}$ & $N A^{a}$ \\
\hline $2005-2009$ & $95(35.6)$ & $377(35.4)$ & $N A^{a}$ & $\mathrm{NA}^{\mathrm{a}}$ \\
\hline $2010-2015$ & $129(48.3)$ & $516(48.5)$ & $N A^{a}$ & $N A^{a}$ \\
\hline \multicolumn{5}{|l|}{ Length of Record Before Index Date (Years) } \\
\hline Mean \pm Std. Dev & $18.5 \pm 4.6$ & $19.4 \pm 4.3$ & 0.96 & $0.93-0.99$ \\
\hline \multicolumn{5}{|l|}{ BMI } \\
\hline$<25 \mathrm{~kg} / \mathrm{m}^{2}$ & $60(22.6)$ & $307(28.9)$ & Reference & Reference \\
\hline $25-30 \mathrm{~kg} / \mathrm{m}^{2}$ & III (4I.7) & $469(44.2)$ & 1.22 & $0.85-1.75$ \\
\hline$>30 \mathrm{~kg} / \mathrm{m}^{2}$ & $75(28.2)$ & $218(20.6)$ & 1.86 & $1.25-2.78$ \\
\hline Unknown & $20(7.5)$ & $67(6.3)$ & 1.61 & $0.88-2.97$ \\
\hline Mean \pm Std. Dev & $26.0 \pm 8.75$ & $25.3 \pm 7.74$ & 1.01 & $1.00-1.03$ \\
\hline \multicolumn{5}{|l|}{ Smoking Status } \\
\hline Non-Smoker & $|2|(45.5)$ & $442(4 I .7)$ & Reference & Reference \\
\hline Current smoker & $27(10.2)$ & $145(13.7)$ & 0.66 & $0.4 I-1.06$ \\
\hline Past-Smoker & $103(38.7)$ & $404(38.1)$ & 0.93 & $0.68-1.26$ \\
\hline Unknown & $15(5.6)$ & $70(6.6)$ & 0.75 & $0.39-1.44$ \\
\hline \multicolumn{5}{|l|}{ Comorbidities at Index Date } \\
\hline Hypertension & $74(27.8)$ & $266(25.1)$ & 1.16 & $0.85-1.60$ \\
\hline Diabetes & $30(11.3)$ & $156(14.7)$ & 0.70 & $0.45-1.09$ \\
\hline Diabetes End Organ Damage & II (4.I) & $43(4.1)$ & 1.01 & $0.49-2.08$ \\
\hline Hyperlipidemia & $40(15.0)$ & $143(13.5)$ & 1.17 & $0.77-1.76$ \\
\hline Alcohol use & $7(2.6)$ & $21(2.0)$ & 1.35 & $0.56-3.23$ \\
\hline Chronic Obstructive Pulmonary Disease & $37(13.9)$ & $109(10.3)$ & 1.46 & $0.96-2.21$ \\
\hline Epilepsy & $8(3.0)$ & $21(2.0)$ & 1.66 & $0.66-4.20$ \\
\hline Autoimmune & $38(14.3)$ & $115(10.8)$ & 1.37 & $0.93-2.04$ \\
\hline Cardiovascular $^{\mathrm{b}}$ & $122(45.9)$ & $395(37.2)$ & 1.53 & I.I4-2.07 \\
\hline Stroke or TIA & $4 \mathrm{I}(15.4)$ & $142(13.4)$ & 1.19 & $0.80-1.76$ \\
\hline Myocardial Infarction & $22(8.3)$ & $102(9.6)$ & 0.83 & $0.5 I-1.37$ \\
\hline Peripheral Vascular Disease & $21(7.9)$ & $64(6.0)$ & 1.34 & $0.79-2.26$ \\
\hline Chronic Heart Failure & $24(9.0)$ & $62(5.8)$ & 1.67 & $1.00-2.80$ \\
\hline Other & $14(5.2)$ & $25(2.4)$ & 1.46 & $0.98-2.20$ \\
\hline Chronic Kidney Disease & $80(30.1)$ & $205(19.3)$ & 2.00 & $1.42-2.79$ \\
\hline Severe CKD & 37 (13.9) & $103(9.7)$ & 1.63 & $1.04-2.55$ \\
\hline Vasculitis & NR & NR & 1.00 & $0.11-8.95$ \\
\hline Coagulopathy & $7(2.6)$ & $18(1.7)$ & 1.62 & $0.65-4.06$ \\
\hline Phlebitis & $31(11.7)$ & $53(5.0)$ & 2.85 & $1.72-4.74$ \\
\hline
\end{tabular}

(Continued) 
Table I (Continued).

\begin{tabular}{|c|c|c|c|c|}
\hline & Cases $(n=266)$ & Controls $(n=1061)$ & OR & $95 \% \mathrm{Cl}$ \\
\hline Dementia & $7(2.6)$ & $20(1.9)$ & 1.37 & $0.57-3.27$ \\
\hline Ulcer & $27(10.2)$ & $96(9.0)$ & 1.16 & $0.72-1.85$ \\
\hline Non-melanoma skin cancer & $22(8.3)$ & $79(7.4)$ & 1.13 & $0.68-1.86$ \\
\hline Prostatectomy & $40(15.0)$ & $143(13.5)$ & 1.16 & $0.77-1.74$ \\
\hline \multicolumn{5}{|c|}{ Charlson Comorbidity Index Score } \\
\hline CCl: I-3 & $108(40.6)$ & $507(47.8)$ & Reference & Reference \\
\hline CCl: 4 & $59(22.2)$ & $225(2 \mid .2)$ & 1.54 & $1.02-2.32$ \\
\hline $\mathrm{CCl}: 5+$ & $99(37.2)$ & $329(31.0)$ & 1.90 & $1.26-2.88$ \\
\hline CCI Score & $4.18 \pm 2.07$ & $3.96 \pm 1.89$ & 1.10 & $1.04-1.17$ \\
\hline \multicolumn{5}{|c|}{ Time Between BPH Diagnosis and Index Date } \\
\hline Less than I year & $33(12.4)$ & $126(11.9)$ & Reference & Reference \\
\hline $1-<2$ years & $25(9.4)$ & $104(9.8)$ & 0.92 & $0.51-1.64$ \\
\hline $2-<5$ years & $72(27.1)$ & $310(29.2)$ & 0.89 & $0.56-1.4 \mid$ \\
\hline $5-<10$ years & $90(33.8)$ & $350(33.0)$ & 0.98 & $0.63-1.54$ \\
\hline$\geq 10$ years & $46(17.3)$ & $17 \mid(16.1)$ & 1.03 & $0.62-1.70$ \\
\hline \multicolumn{5}{|l|}{ Exposure at Index Date } \\
\hline$A B$ only & $169(63.5)$ & $713(67.2)$ & Reference & Reference \\
\hline $5 A R I+A B$ & $33(12.4)$ & $130(12.3)$ & 1.09 & $0.70-1.69$ \\
\hline $5 A R I$ & $64(24.1)$ & $218(20.6)$ & 1.26 & $0.90-1.77$ \\
\hline \multicolumn{5}{|c|}{ Recency of Exposure Closest to Index Date } \\
\hline$A B$ only & $169(63.5)$ & $713(67.2)$ & Reference & Reference \\
\hline Current use of 5ARI & $70(26.3)$ & $250(23.6)$ & 1.21 & $0.86-1.69$ \\
\hline Recent/Past use of 5 ARI & $5(1.9)$ & $21(2.0)$ & 1.02 & $0.38-2.74$ \\
\hline Distant past use of $5 \mathrm{ARI}$ & $22(8.3)$ & $77(7.3)$ & 1.22 & $0.73-2.05$ \\
\hline \multicolumn{5}{|c|}{ Switch Between $A B$ and $A R I$ Before Index Date } \\
\hline No & $183(68.8)$ & $727(68.5)$ & Reference & Reference \\
\hline Yes & $83(31.2)$ & $334(3 \mid .5)$ & 0.99 & $0.73-1.35$ \\
\hline
\end{tabular}

Notes: ${ }^{\mathrm{a} M a t c h i n g}$ factor. ${ }^{\mathrm{b}}$ Cardiovascular disease includes Ischemic heart disease, Acute coronary syndrome, Stroke, pericardial disease, pulmonary hypertension, peripheral vascular disease, chronic heart failure, coronary heart disease.

Abbreviations: OR, odds ratio; $95 \% \mathrm{Cl}, 95 \%$ confidence interval; NA, not applicable; NR, not reportable; Current use, $5 \mathrm{ARI}$ use less than 30 days prior to the index date; Recent use, 5ARI use 3I-II 9 days prior to the index date; Past use, 5ARI use I20-305 days prior to the index date; Distant past use, 5ARI use more than 305 days prior to the index date.

current 5ARI use $1.51(0.98-2.32)$. The increased risk of VTE was higher in patients who had 50 or more 5ARI prescriptions [aOR: $2.29(1.14-4.63)]$. This finding persisted regardless of stratification by various potential confounders, further supporting the finding.

Our study was designed to control for confounding by indication $(\mathrm{BPH})$; we used an active comparator of $\mathrm{AB}$ users rather than a non-exposed referent to control for confounding by severity of BPH. Since men with BPH have increased activity of the 5 alpha reductase enzyme, ${ }^{26}$ they may have a different risk of VTE compared to men without BPH, regardless of treatment; thus, it was important to use an active comparator. However, users of 5ARIs only in this study were less healthy than users of $\mathrm{AB}$ only or of $5 \mathrm{ARI}+\mathrm{AB}$; thus, residual confounding by indication may be present and could explain the elevated risk found in this study. We must also consider, however, the possibility that the active comparator group ( $\mathrm{AB}$ users) also had an increased baseline risk of VTE, and that 5ARI use confers an even stronger risk of VTE than suggested by this study.

Many men in this study were at high risk of VTE, that is, they had multiple comorbidities and cardiovascular risk factors. Our analysis stratified by CCI showed that the risk 
Table 2 Characteristics of Exposed and Unexposed Controls at Index Date: BPH Patients Aged 40-79 in CPRD GOLD I995-20I5

\begin{tabular}{|c|c|c|c|}
\hline & 5ARI Exposed $(n=2 \mid 8)$ & 5ARI+AB Exposed $(n=130)$ & AB Exposed $(n=7 \mid 3)$ \\
\hline \multicolumn{4}{|l|}{ Age at Index Date (years) } \\
\hline$<60$ & $10(4.6)$ & $5(3.9)$ & $67(11.1)$ \\
\hline $60-69$ & $50(22.9)$ & $39(30.0)$ & $148(24.6)$ \\
\hline 70-74 & $34(15.6)$ & $29(22.3)$ & $135(22.4)$ \\
\hline$\geq 75$ & $124(56.9)$ & $57(43.9)$ & $252(4 I .9)$ \\
\hline Mean \pm Std. Dev & $75 . I \pm 8.0$ & $73.6 \pm 7.6$ & $72.5 \pm 8.5$ \\
\hline \multicolumn{4}{|l|}{ Index Year } \\
\hline $1995-1999$ & $6(2.8)$ & NR & $18(2.5)$ \\
\hline $2000-2004$ & $34(15.6)$ & $9(6.9)$ & $105(14.7)$ \\
\hline $2005-2009$ & $77(35.3)$ & $35(26.9)$ & $261(36.6)$ \\
\hline $2010-2015$ & $101(46.3)$ & $86(66.2)$ & $329(46.1)$ \\
\hline \multicolumn{4}{|l|}{ BMI } \\
\hline$<24 \mathrm{~kg} / \mathrm{m}^{2}$ & 74 (33.9) & $28(21.5)$ & $205(28.8)$ \\
\hline $25-30 \mathrm{~kg} / \mathrm{m}^{2}$ & $90(4 \mid .3)$ & $64(49.2)$ & $315(44.2)$ \\
\hline$>30 \mathrm{~kg} / \mathrm{m}^{2}$ & $40(18.4)$ & $33(25.4)$ & $145(20.3)$ \\
\hline Unknown & $14(6.4)$ & $5(3.9)$ & $48(6.7)$ \\
\hline \multicolumn{4}{|l|}{ Smoking Status } \\
\hline Non-Smoker & $92(42.2)$ & $61(46.9)$ & $289(40.5)$ \\
\hline Current smoker & $28(12.8)$ & II (8.5) & $106(14.9)$ \\
\hline Past-Smoker & $80(36.7)$ & $53(40.8)$ & $27 \mid(38.0)$ \\
\hline Unknown & $18(8.3)$ & $5(3.9)$ & $47(6.6)$ \\
\hline \multicolumn{4}{|l|}{ Comorbidities } \\
\hline Hypertension & $49(22.5)$ & $39(30.0)$ & $178(25.0)$ \\
\hline Diabetes & $30(13.8)$ & $21(16.2)$ & $105(14.7)$ \\
\hline End organ damage & $7(3.2)$ & $8(6.2)$ & $28(3.9)$ \\
\hline Hyperlipidemia & $28(12.8)$ & $18(13.8)$ & 97 (13.6) \\
\hline Cardiovascular & $93(42.7)$ & $4 \mid(3 \mid .5)$ & $26 \mid(36.6)$ \\
\hline CVA or TIA & $34(15.6)$ & $15(11.5)$ & $93(13)$ \\
\hline Myocardial Infarction & $29(13.3)$ & II (8.5) & $62(8.7)$ \\
\hline $\mathrm{CHF}$ & $22(7.8)$ & $10(6.1)$ & $54(6.1)$ \\
\hline PVD & $17(7.8)$ & $5(3.8)$ & $42(5.9)$ \\
\hline Alcohol Abuse & $7(3.2)$ & NR & $10(1.4)$ \\
\hline COPD & $22(10.1)$ & $12(9.2)$ & $75(10.5)$ \\
\hline Epilepsy & $7(3.2)$ & NR & $14(2.0)$ \\
\hline Autoimmune & $22(10.1)$ & $12(9.2)$ & $81(11.4)$ \\
\hline CKD & $57(26.1)$ & $25(19.2)$ & $123(17.3)$ \\
\hline Severe CKD & $23(10.6)$ & $22(16.9)$ & $58(8.1)$ \\
\hline Vasculitis & NR & NR & NR \\
\hline Coagulopathy & $N R$ & NR & $12(1.7)$ \\
\hline Phlebitis & $7(3.2)$ & $6(4.6)$ & $40(5.6)$ \\
\hline Prostatectomy & $34(15.6)$ & $15(11.5)$ & 94 (13.2) \\
\hline Dementia & $7(3.2)$ & $3(2.3)$ & $10(1.4)$ \\
\hline Ulcer & $20(9.2)$ & $18(13.8)$ & $58(8.1)$ \\
\hline Non-melanoma skin cancer & $17(7.8)$ & $9(6.9)$ & $53(7.4)$ \\
\hline
\end{tabular}


Table 2 (Continued).

\begin{tabular}{|c|c|c|c|}
\hline & 5ARI Exposed $(n=218)$ & 5ARI+AB Exposed $(n=\mid 30)$ & AB Exposed $(n=7 \mid 3)$ \\
\hline \multicolumn{4}{|c|}{ Charlson Comorbidity Index Score } \\
\hline $\mathrm{CCl}: 1-3$ & $98(45.0)$ & $54(4 I .5)$ & $355(49.8)$ \\
\hline $\mathrm{CCl}: 4$ & $44(20.2)$ & $31(23.9)$ & $150(21.0)$ \\
\hline $\mathrm{CCl}: 5+$ & $76(34.9)$ & $45(34.6)$ & $208(29.2)$ \\
\hline \multicolumn{4}{|c|}{ Time between BPH diagnosis and Index Date } \\
\hline Less than I year & $24(11.0)$ & $14(10.8)$ & $88(12.3)$ \\
\hline $1-<2$ years & $17(7.8)$ & $20(15.4)$ & $67(9.4)$ \\
\hline $2-<5$ years & $66(30.3)$ & $29(22.3)$ & $215(30.2)$ \\
\hline $5-<10$ years & $62(28.4)$ & $46(35.4)$ & $242(33.9)$ \\
\hline$\geq 10$ years & $49(22.5)$ & $21(16.2)$ & $101(14.2)$ \\
\hline
\end{tabular}

Notes: Categorical variables are presented as $\mathrm{N}(\%)$. Continuous variables are presented as Mean (SD).

Abbreviation: NR, not reportable.

of VTE increased with higher comorbidity scores (test for trend $\mathrm{p}=0.01$ ) similar to a previous study, which found that patients with a CCI score of 4 and higher had a higher risk of VTE. ${ }^{38}$ This supports the notion that there may be residual confounding in this study and that higher morbidity could explain the increased risk of VTE in 5ARI users.

To the best of our knowledge, this is the first study to evaluate the risk of VTE in relation to the use of 5ARIs. The closest comparisons available are in studies that evaluated the risk of VTE associated with testosterone use.
A study by Baillargeon et $\mathrm{al}^{39}$ found that testosterone therapy was not associated with an increased risk of VTE in middle-aged and older men with testosterone deficiency. Since 5ARI inhibits the production of DHT, a metabolite of testosterone, we expect 5ARI to have an opposing effect on VTE compared to testosterone therapy, ie an increase in VTE risk.

There is growing evidence that DHT has cardiovascular protective properties and an anti-inflammatory effect that promotes endothelial health, ${ }^{7,40}$ thus with inhibition of

Table 3 Crude and Adjusted Odds Ratios and Distribution of Exposure at Index Date Among Venous Thromboembolism Cases and Matched Controls: BPH Patients Aged 40-79 in CPRD GOLD 1995-20I5

\begin{tabular}{|c|c|c|c|c|}
\hline & Cases $(n=266)$ & Controls $(n=1061)$ & Crude OR & Adjusted* OR \\
\hline \multicolumn{5}{|l|}{ Any Exposure at Index Date } \\
\hline$A B$ only & 169 (63.5\%) & 713 (67.2\%) & Reference & Reference \\
\hline $5 A R I$ & $64(24.1 \%)$ & $218(20.6 \%)$ & $1.26(0.90-1.77)$ & $\mathrm{I} .4 \mathrm{I}(0.97-2.07)$ \\
\hline $5 A R I+A B$ & $33(12.4 \%)$ & $130(12.3 \%)$ & $1.09(0.70-1.69)$ & $\mathrm{I} .27(0.72-2.2 \mathrm{I})$ \\
\hline \multicolumn{5}{|l|}{ Recency by Exposure at Index Date } \\
\hline$A B$ only & 169 (63.5\%) & $713(67.2 \%)$ & Reference & Reference \\
\hline Current use of 5ARI & 44 (16.5\%) & $138(13.0 \%)$ & $1.36(0.92-2.03)$ & $1.51(0.98-2.32)$ \\
\hline Recent/Past/Distant past use of $5 \mathrm{ARI}$ & $20(7.5 \%)$ & $80(7.5 \%)$ & $1.07(0.63-1.82)$ & $1.23(0.70-2.17)$ \\
\hline Current use of $5 A R I+A B$ & $26(9.8 \%)$ & 112 (I0.6\%) & $0.99(0.61-1.62)$ & $1.16(0.64-2.10)$ \\
\hline Recent/Past/Distant past use of $5 A R I+A B$ & $7(2.6 \%)$ & $18(1.7 \%)$ & $1.67(0.67-4.15)$ & $1.93(0.71-5.25)$ \\
\hline
\end{tabular}

Notes: Variables are presented as $\mathrm{N}$ (\%). *Adjusted for cardiovascular disease, chronic kidney disease, switch, body mass index and CCl. Conditional on the matching factors.

Abbreviations: OR, odds ratio; $95 \% \mathrm{CI}, 95 \%$ confidence interval; Current use, 5 ARI use less than 30 days prior to the index date; Recent use, $5 \mathrm{ARI}$ use $3 \mathrm{I}-\mathrm{II}$ (9) days prior to the index date; Past use, 5ARI use 120-305 days prior to the index date; Distant past use, 5ARI use more than 305 days prior to the index date. 
Table 4 Crude and Adjusted Odds Ratios for the Association Between 5ARI Exposure and VTE Stratified by Charlson Comorbidity Index Score; BPH Patients Aged 40-79 in CPRD GOLD 1995-20I5

\begin{tabular}{|c|c|c|c|c|}
\hline & Cases $(n=266)$ & Controls $(n=1061)$ & Crude OR (95\% Cl) & Adjusted* OR (95\% Cl) \\
\hline \multicolumn{5}{|c|}{ All Patients } \\
\hline \multicolumn{5}{|l|}{ Recency of any 5ARI } \\
\hline$A B$ only & $169(63.5)$ & $713(67.2)$ & I.0 (reference) & I.0 (reference) \\
\hline Current use of $5 A R I \pm A B$ & $70(26.3)$ & $250(23.6)$ & $1.21(0.86-1.69)$ & $1.38(0.93-2.06)$ \\
\hline Recent/Past/Distant past use of $5 A R I \pm A B$ & $27(10.2)$ & $98(9.2)$ & I.I8 (0.74-1.88) & $1.37(0.82-2.30)$ \\
\hline \multicolumn{5}{|c|}{ Comorbidity Score: I-3 } \\
\hline \multicolumn{5}{|l|}{ Recency of any $5 A R I$} \\
\hline$A B$ only & $80(74.1)$ & $355(70.0)$ & 1.0 (reference) & I.0 (reference) \\
\hline Current use of $5 A R I \pm A B$ & $16(14.8)$ & $107(2 \mathrm{I} . \mathrm{I})$ & $0.67(0.37-1.19)$ & $0.94(0.48-1.82)$ \\
\hline Recent/Past/Distant past use of $5 A R I \pm A B$ & $12(11.1)$ & $45(8.9)$ & $1.19(0.60-2.35)$ & $1.49(0.72-3.08)$ \\
\hline \multicolumn{5}{|c|}{ Comorbidity Score: 4} \\
\hline \multicolumn{5}{|l|}{ Recency of any 5ARI } \\
\hline$A B$ only & $35(59.3)$ & $150(66.7)$ & I.0 (reference) & I.0 (reference) \\
\hline Current use of $5 A R I \pm A B$ & $17(28.8)$ & $57(25.5)$ & $1.27(0.66-2.44)$ & $1.53(0.7 \mathrm{I}-3.28)$ \\
\hline Recent/Past/Distant past use of $5 A R I \pm A B$ & $7(11.9)$ & $18(8.0)$ & $1.66(0.64-4.27)$ & $1.62(0.55-4.73)$ \\
\hline \multicolumn{5}{|c|}{ Comorbidity Score: 5} \\
\hline \multicolumn{5}{|l|}{ Recency of any 5ARI } \\
\hline$A B$ only & $54(54.6)$ & $208(63.2)$ & I.0 (reference) & I.0 (reference) \\
\hline Current use of $5 A R I \pm A B$ & $37(37.4)$ & $86(26.1)$ & $1.66(1.02-2.70)$ & $1.57(0.90-2.75)$ \\
\hline Recent/Past/Distant past use of $5 A R I \pm A B$ & $8(8.1)$ & $35(10.6)$ & $0.88(0.39-2.01)$ & $0.75(0.31-1.80)$ \\
\hline
\end{tabular}

Notes: Variables are presented as $\mathrm{N}$ (\%). *Adjusted for age, cardiovascular disease, chronic kidney disease, switch, and body mass index. Conditional on the matching factors.

Abbreviations: OR, odds ratio; $95 \% \mathrm{Cl}, 95 \%$ confidence interval; Current use, $5 \mathrm{ARI}$ use less than 30 days prior to the index date; Recent use, $5 \mathrm{ARI}$ use $3 \mathrm{I}-\mathrm{II} 9$ days prior to the index date; Past use, 5ARI use 120-305 days prior to the index date; Distant past use, 5ARI use more than 305 days prior to the index date.

DHT production (such as is seen with 5ARI use), an increase in VTE risk among 5ARI users compared to nonusers is biologically plausible.

While the mechanisms for developing VTE and atherothrombotic outcomes are not exactly the same, some parallels can be drawn from studies that evaluated the risk of cerebrovascular disease, MI, and strokes. A previous study showed that androgen-deprivation therapies (ADTs) such as gonadotropin-releasing hormone agonists and orchiectomy, which reduce the serum levels of testosterone and DHT, increase the risk of MI and stroke. ${ }^{41,42}$ Similarly, a study by Yeap et $\mathrm{al}^{43}$ showed that higher DHT levels were associated with a decreased risk of IHD mortality and optimal testosterone levels were associated with a reduced risk of all-cause mortality. However, studies examining the relationship of 5ARI use to cardiovascular outcomes have had conflicting findings. ${ }^{5,6,43-49}$
One of the arguments against a causal explanation for the increased risk of VTE with 5ARI use in previous studies is that ADTs that cause harmful metabolic effects and increase thrombotic events tend to suppress a broad spectrum of androgens. Since 5ARIs only suppress DHT, they cannot significantly increase cardiovascular risk through this mechanism. ${ }^{41,50}$ In this study, we found an increased risk with heavy use of 5ARIs, perhaps because the suppression of DHT with low-dose 5ARI use is not sufficient to cause harmful thrombotic effects, while at higher cumulative dose, it may activate prothrombotic pathways that are comparable to other ADTs. BPH patients have high levels of DHT, which is corrected to the physiologic range with 5ARI use. ${ }^{51-53}$ With higher doses, further suppression of DHT levels may have a negative effect. This may explain why the users of 5ARIs with $>50$ prescriptions or $>10,000 \mathrm{mcg}$ of 5ARI 
Table 5 Crude and Adjusted Odds Ratios and Distribution of Exposure at Index Date by Number of Prescriptions and Cumulative Dose (Mcg) of 5ARI Among Venous Thromboembolism Cases and Matched Controls: BPH Patients Aged 40-79 in CPRD GOLD 1995-2015

\begin{tabular}{|c|c|c|c|c|}
\hline & Cases $(n=266)$ & Controls $(n=1061)$ & Crude OR & Adjusted* OR \\
\hline \multicolumn{5}{|c|}{ Number of Prescriptions by Timing at Index Date } \\
\hline$A B$ only & $169(63.5 \%)$ & $713(67.2 \%)$ & Reference & Reference \\
\hline \multicolumn{5}{|l|}{$5 A R I$} \\
\hline Current I-24 & $20(7.5 \%)$ & $64(6.1 \%)$ & $1.32(0.77-2.29)$ & $1.50(0.84-2.69)$ \\
\hline Current 25-49 & $8(3.0 \%)$ & $36(3.4 \%)$ & $0.89(0.39-2.04)$ & $0.85(0.36-2.04)$ \\
\hline Current 50+ & $16(6.0 \%)$ & $38(3.6 \%)$ & $1.78(0.97-3.26)$ & $2.29(1.14-4.63)$ \\
\hline Recent/Past/Distant past use & $20(7.5 \%)$ & $80(7.5 \%)$ & $1.07(0.63-1.82)$ & $1.23(0.70-2.18)$ \\
\hline \multicolumn{5}{|l|}{$5 A R I+A B$} \\
\hline Current I-24 & $8(3.0 \%)$ & $60(5.7 \%)$ & $0.52(0.23-1.18)$ & $0.62(0.25-1.52)$ \\
\hline Current 25-49 & $10(3.8 \%)$ & $26(2.5 \%)$ & $1.74(0.79-3.83)$ & $1.96(0.82-4.67)$ \\
\hline Current $50+$ & $8(3.0 \%)$ & $26(2.5 \%)$ & $1.30(0.58-2.92)$ & $1.65(0.64-4.26)$ \\
\hline Recent/Past/Distant past use & $7(2.6 \%)$ & $18(1.7 \%)$ & $1.75(0.70-4.35)$ & $2.03(0.74-5.56)$ \\
\hline \multicolumn{5}{|c|}{ Cumulative Dose of Prescriptions (mcgs) by Timing at Index Date } \\
\hline$A B$ only & $169(63.5 \%)$ & $713(67.2 \%)$ & Reference & Reference \\
\hline \multicolumn{5}{|l|}{$5 A R I$} \\
\hline Current I-999 & $5(1.9 \%)$ & $19(1.8 \%)$ & $1.13(0.38-3.36)$ & $1.32(0.43-4.05)$ \\
\hline Current 1000-9999 & $17(6.4 \%)$ & $70(6.6 \%)$ & $0.99(0.56-1.76)$ & $1.09(0.60-1.98)$ \\
\hline Current $10,000+$ & $22(8.3 \%)$ & $49(4.6 \%)$ & $1.95(I .12-3.4 I)$ & $2.15(1.18-3.92)$ \\
\hline Recent/Past/Distant past use & $20(7.5 \%)$ & $80(7.5 \%)$ & $1.06(0.62-1.80)$ & $1.22(0.69-2.16)$ \\
\hline \multicolumn{5}{|l|}{$5 A R I+A B$} \\
\hline Current I-999 & $5(1.9 \%)$ & $5(0.5 \%)$ & $4.03(1.16-14.00)$ & $5.47(1.45-20.58)$ \\
\hline Current 1000-9999 & $13(4.9 \%)$ & 52 (4.9\%) & $1.09(0.56-2.12)$ & $1.22(0.58-2.57)$ \\
\hline Current $10,000+$ & $8(3.0 \%)$ & 55 (5.2\%) & $0.58(0.26-1.30)$ & $0.67(0.27-1.64)$ \\
\hline Recent/Past/Distant past use & $7(2.6 \%)$ & 18 (1.7\%) & $1.66(0.67-4.14)$ & $1.90(0.70-5.19)$ \\
\hline
\end{tabular}

Notes: Variables are presented as $\mathrm{N}$ (\%). *Adjusted for cardiovascular disease, chronic kidney disease, switch, body mass index and CCl. Conditional on the matching factors.

Abbreviations: OR, odds ratio; $95 \% \mathrm{CI}, 95 \%$ confidence interval; current use, 5ARI use less than 30 days prior to the index date; Recent use, 5ARI use $3 \mathrm{I}-\mathrm{II} 9$ days prior to the index date; Past use, 5ARI use 120-305 days prior to the index date; Distant past use, 5ARI use more than 305 days prior to the index date.

in our study were at an increased risk of VTE, while in patients at lower cumulative doses/number of prescriptions, there was a near null effect.

Our study has several strengths. First, we used a longitudinal primary care database with high accuracy of diagnoses and completeness of drug prescription data. We found known risk factors to be independently associated with the risk of VTE (ie, history of fractures, surgeries, cardiovascular comorbidities, phlebitis, CKD, extended hospitalizations), providing confidence in the quality of the data and its ability to detect associations between BPH treatments and risk of VTE. Second, we controlled our analyses for a range of potential confounders, including age, BMI, cardiovascular disease, CKD and others, using the CCI score. Since information on diseases and drug exposures was recorded in the absence of a study hypothesis, there is no risk of recall bias. By excluding men who had insufficient history in their medical record before study start in both cases and controls, we reduced the risk of including men with recurrent, rather than incident, VTE. Furthermore, the mean record length before the index date in this study was greater than 18 years enabling us to assess the potential risks and benefits of long-term drug use.

There are some limitations to consider. We did not have access to information regarding medication adherence because drug information in the CPRD reflects written, not 
pharmacy dispensed or used prescriptions. However, patients with repeat $(2+)$ prescriptions are more likely to have filled and used the medications and the risk of VTE was higher in these patients, suggesting that non-use of prescribed medications did not bias the results.

Objective measures of BPH severity are not captured in the CPRD GOLD data, beyond the duration of BPH. Therefore, we were unable to assess the impact of BPH severity on the results. However, we minimized the heterogeneity of $\mathrm{BPH}$ severity by only including patients with $5 \mathrm{ARI}$ and/or $\mathrm{AB}$ prescriptions. Our study results suggest that the duration of $\mathrm{BPH}$ is not an independent risk factor for VTE and that stratification by BPH duration does not change the interpretation of our findings. We considered whether patients receiving both ABs and 5ARIs instead of one or the other could be at higher risk of VTE, but our findings did not support this.

In conclusion, our study provides evidence that high cumulative dose of 5ARIs may increase the risk of incident VTE in men with BPH who are free of major VTE risk factors (eg cancers, fractures, surgeries and strong cardiovascular risk factors), potentially due to excessive inhibition of the anti-inflammatory properties of DHT in promoting endothelial health and preventing thrombogenesis.

Since BPH is a common condition in men and 5ARIs are primary drug treatments, further studies of 5ARI use are warranted to inform use in every day clinical practice.

\section{Disclosure}

Dr David McManus reports grants, personal fees from Bristol Myers Squibb, grants, personal fees from Pfizer, grants from Boehringer Ingelheim, personal fees from Flexcon, non-financial support from Apple, personal fees from Avania, grants from Philips, personal fees, nonfinancial support from Fitbit, grants from Heart Rhythm Society, non-financial support from Samsung, personal fees from Rose Consulting, outside the submitted work. The authors report no other conflicts of interest in this work.

\section{References}

1. Naslund MJ, Gilsenan AW, Midkiff KD, Bown A, Wolford ET, Wang J. Prevalence of lower urinary tract symptoms and prostate enlargement in the primary care setting. Int J Clin Pract. 2007;61 (9):1437-1445. doi:10.1111/j.1742-1241.2007.01508.x

2. Emberton M, Marberger M, De La Rosette J. Understanding patient and physician perceptions of benign prostatic hyperplasia in Europe: the prostate research on behaviour and education (PROBE) survey. Int J Clin Pract. 2008;62:18. doi:10.1111/j.1742-1241.2007.01635.x

3. Emberton M, Fitzpatrick JM, Rees J. Risk stratification for benign prostatic hyperplasia (BPH) treatment. BJU Int. 2011;107:876-880. doi:10.1111/j.1464-410X.2010.10041.x
4. Saylor PJ, Smith MR. Metabolic complications of androgen deprivation therapy for prostate cancer. J Urol. 2009;181(5):1998-2008. doi:10.1016/j.juro.2009.01.047

5. Keating NL, O'Malley AJ, Freedland SJ, Smith MR. Diabetes and cardiovascular disease during androgen deprivation therapy: observational study of veterans with prostate cancer. J Natl Cancer Inst. 2010;102(1):39-46. doi:10.1093/jnci/djp404

6. Jespersen CG, Nørgaard M, Borre M. Androgen-deprivation therapy in treatment of prostate cancer and risk of myocardial infarction and stroke: a nationwide Danish population-based cohort study. Eur Urol. 2014;65(4):704-709. doi:10.1016/j.eururo.2013.02.002

7. Araujo AB, Kupelian V, Page ST, Handelsman DJ, Bremner WJ, McKinlay JB. Sex steroids and all-cause and cause-specific mortality in men. Arch Intern Med. 2007;167:1252. doi:10.1001/ archinte.167.12.1252

8. Walker RF, Zakai NA, Maclehose RF, et al. Association of testosterone therapy with risk of venous thromboembolism among men with and without hypogonadism. JAMA Intern Med. 2020;180 (2):190-197. doi:10.1001/jamainternmed.2019.5135

9. Yao S, Till C, Kristal AR, et al. Serum estrogen levels and prostate cancer risk in the prostate cancer prevention trial: a nested case-control study. Cancer Causes Control. 2011;22:1121-1131. doi:10.1007/s10552-011-9787-7

10. Simon T, De Jonage-canonico MBY, Oger E, et al. Indicators of lifetime endogenous estrogen exposure and risk of venous thromboembolism. J Thromb Haemost. 2006;4:71-76. doi:10.1111/ j.1538-7836.2005.01693.x

11. Choi BK, Cheon K, Cho B-H, Jung JW, Y1 K. Cerebral venous sinus thrombosis associated with dutasteride use. Yonsei Med J. 2020;61 (6):553-555. doi:10.3349/ymj.2020.61.6.553

12. Kang DW, Jeong HG, Kim HR, et al. Finasteride induced cerebral venous thrombosis. J Korean Neurol Assoc. 2015;33:238-240. doi:10.17340/jkna.2015.3.22

13. Mansouri P, Farshi S, Safar F. Finasteride-induced gynecomastia. Indian J Dermatol Venereol Leprol. 2009;75:309-310. doi:10.4103/ 0378-6323.51273

14. Ferrando J. Unilateral gynecomastia induced by treatment with $1 \mathrm{mg}$ of oral finasteride. Arch Dermatol. 2004. doi:10.1001/ archderm.138.4.543

15. Green L, Wysowski DK, Fourcroy JL. Gynecomastia and breast cancer during finasteride therapy. $N$ Engl J Med. 2002;138:543. doi:10.1056/nejm199609123351116

16. Roehrborn CG, Boyle P, Nickel JC, Hoefner K, Andriole G. Efficacy and safety of a dual inhibitor of 5-alpha-reductase types 1 and 2 (dutasteride) in men with benign prostatic hyperplasia. Urology. 2002;60(3):434-441. doi:10.1016/S0090-4295(02)01905-2

17. Desgrandchamps F, Droupy S, Irani J, Saussine C, Comenducci A. Effect of dutasteride on the symptoms of benign prostatic hyperplasia, and patient quality of life and discomfort, in clinical practice. BJU Int. 2006;98(1):83-88. doi:10.1111/j.1464-410X.2006.06241.x

18. Debruyne F, Barkin J, Van EP, Reis M, Tammela TLJ, Roehrborn C. Efficacy and safety of long-term treatment with the dual $5 \alpha$-reductase inhibitor dutasteride in men with symptomatic benign prostatic hyperplasia. Eur Urol. 2004;46(4):488-495. doi:10.1016/j. eururo.2004.05.008

19. Gormley GJ, Stoner E, Bruskewitz RC, et al. The effect of finasteride in men with benign prostatic hyperplasia. 1992. J Urol. 2002;167(2 Pt 2):1102-1107. doi:10.1016/s0022-5347(02)80349-4

20. Andriole GL, Kirby R. Safety and tolerability of the dual $5 \alpha$ reductase inhibitor dutasteride in the treatment of benign prostatic hyperplasia. Eur Urol. 2003;44(1):82-88. doi:10.1016/S03022838(03)00198-2

21. Gregson J, Kaptoge S, Bolton T, et al. Cardiovascular risk factors associated with venous thromboembolism. JAMA Cardiol. 2019;4 (2):163. doi:10.1001/jamacardio.2018.4537 
22. Thompson IM, Goodman PJ, Tangen CM, et al. The influence of finasteride on the development of prostate cancer. $N$ Engl $J$ Med. 2003;349(3):215-224. doi:10.1056/nejmoa030660

23. Miller JA, Pramanik B, Gilhooly P. Waxing and waning gynecomastia: an indication of noncompliant use of prescribed medication. South Med J. 1999;92(6):615-617. doi:10.1097/00007611199906000-00012

24. Olsen EA, Hordinsky M, Whiting D, et al. The importance of dual $5 \alpha$-reductase inhibition in the treatment of male pattern hair loss: results of a randomized placebo-controlled study of dutasteride versus finasteride. J Am Acad Dermatol. 2006;55(6):1014-1023. doi:10.1016/j.jaad.2006.05.007

25. Grino P, Stoner E, Andriole GL, et al. Finasteride for the treatment and control of benign prostatic hyperplasia: summary of Phase III controlled studies. Eur Urol. 1994;25(SUPPL. 1):24-28. doi:10.1159/ 000475328

26. Traish AM, Hassani J, Guay AT, Zitzmann M, Hansen ML. Adverse side effects of $5 \alpha$-reductase inhibitors therapy: persistent diminished libido and erectile dysfunction and depression in a subset of patients. J Sex Med. 2011;8(3):872-884. doi:10.1111/j.1743-6109.2010.02157.x

27. Shenoy NK, Prabhakar SM. Finasteride and male breast cancer: does the MHRA report show a link? J Cutan Aesthet Surg. 2010;3 (2):102-105. doi:10.4103/0974-2077.69022

28. Tsuji Y, Nakayama T, Bono K, Kitamura M, Imafuku I. Two cases of stroke associated with the use of finasteride, an approved drug for male-pattern hair loss in Japan. Rinsho Shinkeigaku. 2014;54:423-428. doi:10.5692/clinicalneurol.54.423

29. Sacco S, Ricci S, Degan D, Carolei A. Migraine in women: the role of hormones and their impact on vascular diseases. J Headache Pain. 2012;13(3):177-189. doi:10.1007/s10194-012-0424-y

30. Rosendaal FR, Helmerhorst FM, Vandenbroucke JP. Female hormones and thrombosis. Arterioscler Thromb Vasc Biol. 2002;22 (2):201-210. doi:10.1161/hq0202.102318

31. Parkin L, Sharples K, Hernandez RK, Jick SS. Risk of venous thromboembolism in users of oral contraceptives containing drospirenone or levonorgestrel: nested case-control study based on UK general practice research database. BMJ. 2011;342(apr21 2):d2139d2139. doi:10.1136/bmj.d2139

32. Malkin CJ, Pugh PJ, Jones RD, Jones TH, Channer KS. Testosterone as a protective factor against atherosclerosis - immunomodulation and influence upon plaque development and stability. J Endocrinol. 2003;178:373-380. doi:10.1677/joe.0.1780373

33. García Rodríguez LA, Pérez Gutthann S. Use of the UK general practice research database for pharmacoepidemiology. $\mathrm{Br} J$ Clin Pharmacol. 1998;45(5):419-425. doi:10.1046/j.13652125.1998.00701.x

34. Lawrenson R, Todd JC, Leydon GM, Williams TJ, Farmer RDT. Validation of the diagnosis of venous thromboembolism in general practice database studies. Br J Clin Pharmacol. 2000;49:591-596. doi:10.1046/j.1365-2125.2000.00199.x

35. Huerta C, Johansson S, Wallander MA, García Rodríguez LA. Risk factors and short-term mortality of venous thromboembolism diagnosed in the primary care setting in the United Kingdom. Arch Intern Med. 2007;167:935. doi:10.1001/archinte.167.9.935

36. Charlson ME, Szatrowski TP, Peterson J, Gold J. Validation of a combined comorbidity index. J Clin Epidemiol. 1994;47(11):12451251. doi:10.1016/0895-4356(94)90129-5

37. Charlson ME, Pompei P, Ales KL, MacKenzie CR. A new method of classifying prognostic comorbidity in longitudinal studies: development and validation. J Chronic Dis. 1987;40:373-383. doi:10.1016/ 0021-9681(87)90171-8

38. Ording AG, Horváth-Puhõ E, Lash TL, et al. Prostate cancer, comorbidity, and the risk of venous thromboembolism: a cohort study of 44,035 Danish prostate cancer patients,1995-2011.Cancer.2015;121 (20):3692-3699. doi:10.1002/cncr.29535
39. Baillargeon J, Urban RJ, Morgentaler A, et al. Risk of venous thromboembolism in men receiving testosterone therapy. Mayo Clin Proc. 2015;90:1038-1045. doi:10.1016/j.mayocp.2015.05.012

40. Swerdloff RS, Dudley RE, Page ST, Wang C, Salameh WA. Dihydrotestosterone: biochemistry, physiology, and clinical implications of elevated blood levels. Endocr Rev. 2017;38:220. doi:10.1210/ er.2016-1067

41. Nishiyama T, Hashimoto Y, Takahashi K. The influence of androgen deprivation therapy on dihydrotestosterone levels in the prostatic tissue of patients with prostate cancer. Clin Cancer Res. 2004;10 (21):7121-7126. doi:10.1158/1078-0432.CCR-04-0913

42. Chang J, Choi S, Kim K, Park SM. Cardiovascular Safety and Possible Benefit of a 5-Alpha Reductase Inhibitor among Benign Prostatic Hyperplasia Patients, A Nationally Representative Cohort of Korean Men. J Clin Med. 2019;8(5):733. doi:10.3390/jcm8050733

43. Yeap BB, Alfonso H, Paul Chubb SA, et al. In older men an optimal plasma testosterone is associated with reduced all-cause mortality and higher dihydrotestosterone with reduced ischemic heart disease mortality, while estradiol levels do not predict mortality. $J$ Clin Endocrinol Metab. 2014;99(1):E9-E18. doi:10.1210/jc.2013-3272

44. Azzouni F, Godoy A, Li Y, Mohler J. The 5 alpha-reductase isozyme family: a review of basic biology and their role in human diseases. Adv Urol. 2012;2012:1-18. doi:10.1155/2012/530121

45. Roehrborn CG, Marks LS, Fenter T, et al. Efficacy and safety of dutasteride in the four-year treatment of men with benign prostatic hyperplasia. Urology. 2004;63(4):709-715. doi:10.1016/j. urology.2004.01.001

46. Trost L, Saitz TR, Hellstrom WJ. Side effects of 5-alpha reductase inhibitors: a comprehensive review. Sex Med Rev. 2013;1:24-41. doi:10.1002/smrj.3

47. Shores MM, Biggs ML, Arnold AM, et al. Testosterone, dihydrotestosterone, and incident cardiovascular disease and mortality in the cardiovascular health study. J Clin Endocrinol Metab. 2014;99 (6):2061-2068. doi:10.1210/jc.2013-3576

48. Loke YK, Ho R, Smith M, et al. Systematic review evaluating cardiovascular events of the 5-alpha reductase inhibitor Dutasteride. J Clin Pharm Ther. 2013;38(5):405-415. doi:10.1111/ jcpt. 12080

49. Hsieh TF, Yang YW, Sen LS, et al. Use of 5-alpha-reductase inhibitors did not increase the risk of cardiovascular diseases in patients with benign prostate hyperplasia: a five-year follow-up study. PLoS One. 2015;10:e0119694. doi:10.1371/journal.pone.0119694

50. Klotz L, Nabid A, Higano C, Ryan C, Kebabdjian M, Chin J. Effect of dutasteride in men receiving intermittent androgen ablation therapy: the AVIAS trial. J Can Urol Assoc. 2014;8(11-12):789. doi: 10.5489 /cuaj. 2332

51. Ghanadian R, Lewis JG, Chisolm GD, O’Donoghue EPN. Serum dihydrotestosterone in patients with benign prostatic hypertrophy. Br J Urol. 1977;49:541-544. doi:10.1111/j.1464-410X.1977. tb04202.x

52. Horton R, Hsieh P, Barberia J, Pages L, Cosgrove M. Altered blood androgens in elderly men with prostate hyperplasia. $J$ Clin Endocrinol Metab. 1975;41:793-796. doi:10.1210/jcem-41-4-793

53. Uygur MC, Arik AI, Altuğ U, Erol D. Effects of the $5 \alpha$-reductase inhibitor finasteride on serum levels of gonadal, adrenal, and hypophyseal hormones and its clinical significance: a prospective clinical study. Steroids. 1998;63:208-213. doi:10.1016/S0039-128X(98) $00005-1$ 


\section{Publish your work in this journal}

Clinical Epidemiology is an international, peer-reviewed, open access, online journal focusing on disease and drug epidemiology, identification of risk factors and screening procedures to develop optimal preventative initiatives and programs. Specific topics include: diagnosis, prognosis, treatment, screening, prevention, risk factor modification,

Submit your manuscript here: https://www.dovepress.com/clinical-epidemiology-journal systematic reviews, risk \& safety of medical interventions, epidemiology \& biostatistical methods, and evaluation of guidelines, translational medicine, health policies \& economic evaluations. The manuscript management system is completely online and includes a very quick and fair peer-review system, which is all easy to use. 